\title{
A Six-Year Investigation of the Dynamics of Avirulence Allele Profiles, Blackleg Incidence, and Mating Type Alleles of Leptosphaeria maculans Populations Associated with Canola Crops in Manitoba, Canada
}

\author{
W. G. Dilantha Fernando ${ }^{\dagger}$, Xuehua Zhang, Carrie Selin, Zhongwei Zou, and Sakaria H. Liban, Department of Plant Science, University \\ of Manitoba, Winnipeg, MB, R3T 2N2, Canada; Debra L. McLaren, Agriculture and Agri-Food Canada, Brandon Research and Development \\ Centre, Brandon, MB, R7A 5Y3, Canada; Anastasia Kubinec, Crops Branch - Industry Development, Manitoba Agriculture, Carman, MB, \\ R0G 0J0, Canada; and Paula S. Parks, M. Harunur Rashid, K. Rasanie E. Padmathilake, Lihua Rong, Cunchun Yang, Belaghihalli \\ N. Gnanesh, and Shuanglong Huang, Department of Plant Science, University of Manitoba, Winnipeg, MB, R3T 2N2, Canada
}

\begin{abstract}
Blackleg, caused by the fungal pathogen Leptosphaeria maculans, is one of the most economically important diseases of canola (Brassica napus, oilseed rape) worldwide. This study assessed incidence of blackleg, the avirulence allele, and mating type distributions of $L$. maculans isolates collected in commercial canola fields in Manitoba, Canada, from 2010 to 2015. A total of $956 \mathrm{~L}$. maculans isolates were collected from 2010 to 2015 to determine the presence of 12 avirulence alleles using differential canola cultivars and/or PCR assays specific for each avirulence allele. AvrLm2, AvrLm4, AvrLm5, AvrLm6, AvrLm7, AvrLm11, and AvrLmS

were detected at frequencies ranging from 97 to $33 \%$, where the AvrLm1, AvrLm3, AvrLm 9, AvrLepR1, and AvrLepR2 alleles were the least abundant. When the race structure was examined, a total of 170 races were identified among the 956 isolates, with three major races, AvrLm-2-4 5-6-7-11, AvrLm-2-4-5-6-7-11-S, and Avr-1-4-5-6-7-11-(S) accounting for 15,10 , and $6 \%$ of the total fungal population, respectively. The distribution of the mating type alleles (MAT1-1 and MAT1-2) indicated that sexual reproduction was not inhibited in any of the nine Manitoba regions in any of the years L. maculans isolates were collected.
\end{abstract}

Blackleg, caused by the fungal pathogen Leptosphaeria maculans, has been identified as one of the major diseases affecting production in many canola (oilseed rape, Brassica napus) growing regions worldwide (Fitt et al. 2006). This disease can be controlled by the utilization of resistant varieties, crop rotations, and fungicide applications (Kutcher et al. 2013). Both qualitative ( $R$-gene resistance) resistance and quantitative (adult plant resistance, APR) have been identified in Brassica species (Balesdent et al. 2001; Delourme et al. 2006; Jestin et al. 2011; Rimmer 2006). Prior genetic studies indicated a gene for gene relationship between an $R$ gene and the corresponding avirulence $(A v r)$ gene for this pathogen (Ansan-Melayah et al. 1998; Balesdent et al. 2001). Using $R$ gene-mediated resistance is often a very effective strategy for disease control when the fungal populations harbor a high frequency of the corresponding avirulence alleles (Daverdin et al. 2012). However, intensive use of single $R$ genes will exert selection pressure on the fungal populations, and can result in pathogen adaptation to $R$ genes in the host plants. Previous studies indicated that the pathogen can adapt to the selection pressure within a few years under both experimental conditions and commercial agricultural systems (Zhang et al. 2016). In experimental conditions, $R \operatorname{lm} 6$ resistance was not effective after three growing seasons (Brun et al. 2010). In France, Rlml resistance was overcome by the fungal populations within three growing seasons (Rouxel et al. 2003). Similarly, LepR3 resistance in Australia lost effect a few years after the release of a commercial LepR3-carrying variety (Sprague et al. 2006; Van de Wouw et al. 2010, 2014). In western Canada, outbreaks of blackleg in crops of commercial canola varieties, carrying blackleg resistance genes, has been recorded

${ }^{\dagger}$ Corresponding author: W. G. D. Fernando;

E-mail: Dilantha.Fernando@umanitoba.ca

The authors gratefully acknowledge the operational funding support for this research provided by Canola Council of Canada's CARP Program, SaskCanola and a NSERC Discovery Grant to WGDF.

Accepted for publication 9 November 2017.

(C) 2018 The American Phytopathological Society frequently since 2007 (Chen and Fernando 2006; W. G. D. Fernando, unpublished data). More recently, the breakdown of $R \operatorname{lm} 3$ resistance was reported in western Canada due to intensive use of Rlm3-carrying canola varieties (Zhang et al. 2016).

Avirulence genes of $L$. maculans are usually located in AT-rich, gene-poor regions of the genome (Rouxel et al. 2011). These regions comprise repetitive DNA derived from transposable elements (TEs) and, therefore, provide an unstable genomic environment that promotes rapid gain and loss of avirulence genes (Soyer et al. 2014). For example, deletions, amino acid substitutions, and repeat-induced point (RIP) mutations of AvrLml were observed in L. maculans isolates collected before and after the breakdown of 'sylvestris' resistance in Australia (Van de Wouw et al. 2010). To date, at least 14 Avr genes have been identified in L. maculans, and seven of them have been cloned, including AvrLm1 (Gout et al. 2006b), AvrLm2 (Ghanbarnia et al. 2015), AvrLm3 (Plissonneau et al. 2016), AvrLm5/AvrLmJ1 (Van de Wouw et al. 2014; Balesdent and Howlett, unpublished data), AvrLm4-7 (Parlange et al. 2009), AvrLm6 (Fudal et al. 2007), and AvrLm11 (Balesdent et al. 2013). More than $18 R$ genes in Brassica species have been identified to date: Rlm1 to Rlm11, LepR1 to LepR4, RlmS, BLMR1, and BLMR2 (Balesdent et al. 2013; Eber et al. 2011; Long et al. 2011; Rimmer 2006; Van de Wouw et al. 2009). Only two $R$ genes, LepR3 (which interacts with AvrLm1) and Rlm2 (which interacts with AvrLm2) have been cloned (Larkan et al. 2013, 2015).

Frequent monitoring of fungal populations can provide relevant data that can be used as part of an effective blackleg control strategy. Reports on L. maculans populations in France and North America have provided useful knowledge for manipulating the planting of different resistant varieties with different $R$-genes for control of blackleg (Balesdent et al. 2006; Dilmaghani et al. 2009; Liban et al. 2016; Rouxel et al. 2003). However, there is an increasing demand to monitor long-term dynamics of populations of $L$. maculans due to the high evolutionary potential of the pathogen. Identification of avirulence alleles in L. maculans populations can be achieved via pathogenicity tests using a set of differential varieties with known $R$ genes and/or polymerase chain reaction (PCR) assays specific for cloned avirulence genes. Although pathogenicity tests are labor-intensive and time-consuming, the results are generally very reliable. PCR assays are a rapid and high-throughput alternative for determining the presence of avirulence alleles. However, PCR markers for avirulence alleles need 
to be validated to confirm that the phenotypic results match genotypic data to account for misleading genetic effects such as partial gene deletions, mutations, allelic variants, and gene interactions. The need for marker validation was shown by the gene masking or 'camouflage' effect that AvrLm-4-7 has on the AvrLm3 allele, where the AvrLm3 gene is not recognized by host $R \operatorname{lm} 3$ when AvrLm4-7 is also present and a virulent phenotype occurs (Plissonneau et al. 2016).

The life cycle of $L$. maculans typically involves annual sexual reproduction by production of ascospores, and asexual reproduction by production of pycnidiospores (Gout et al. 2006a). Sexual reproduction is extremely important for producing primary inoculum via ascospores, and generating genetic variations in fungal populations. L. maculans has two idiomorphs of a single mating type (MAT) locus, MAT1-1 and MAT1-2 (Cozijnsen and Howlett 2003). Earlier studies inferred a lack of sexual reproduction in L. maculans populations in western Canada since pycnidiospores were observed as the primary inoculum source (Dilmaghani et al. 2009; Ghanbarnia et al. 2011).

Annual disease surveys conducted in Manitoba, a province in the Canadian prairies, have reported the incidence of blackleg each year. However, these surveys have not provided information on the Avr alleles and pathogen strains/populations in different regions of Manitoba. This type of data would inform local growers on which varieties are most resistant to pathogen strains in various regions of the province. The objectives of this study were to evaluate $A v r$ allele profiles in Manitoba from 2010 to 2015, blackleg disease incidence, mating type alleles, and changes in avirulence alleles of these $L$. maculans isolates by region and across all areas of the province surveyed. Knowledge of Avr allele frequencies will provide guidance for blackleg resistance breeding and facilitate optimal use of available $R$ genes for growers.

\section{Materials and Methods}

Sample collection. Blackleg infected canola stems were collected from commercial canola fields across Manitoba from 2010 to 2015 as part of the Canadian Plant Disease Survey program (http://phytopath. ca/publication/cpds/). All samples were collected in August when symptoms of blackleg were visible on stems, including the stem base (basal canker). The number of canola fields from which canola basal stem samples were collected were 103 in 2010, 81 in 2011, 108 in 2012, 106 in 2013, 127 in 2014, and 112 in 2015. Fields were distributed across nine regions defined by township as shown in Figure 1. The nine regions were depicted with the following letters and the number of fields within each of the regions indicated in brackets: A, Steinbach area (17 fields); B, Selkirk area (20 fields); C, Swan Valley (18 fields); D, Dauphin (39 fields); E, Central Plains (33 fields); F, Pembina Valley (101 fields); G, Roblin, Russell, Rossburn area (49 fields); H, Southwest area (40 fields); I, and Brandon area (42 fields). A total of 956 L. maculans isolates were obtained from blackleg infected stems: 139 isolates from stems collected in 68 fields in 2010, 61 isolates from 55 fields in 2011, 112 isolates from 54 fields in 2012, 143 isolates from 74 fields in 2013, 207 isolates from 97 fields in 2014, and 302 isolates from 71 fields in 2015.

Determination of blackleg incidence and severity. The incidence of blackleg (disease incidence) in each field was evaluated based on the percentage of blackleg infected stems (stems with basal canker) in 100 plants sampled in each field per year. The percent range of blackleg incidence was tabulated for each region/year. Mean value and standard deviation were not determined because samples collected within each of the nine regions were not collected from the same field (i.e., single replicate) each year due to crop rotation of canola by farmers. Disease severity of each plant (total of 100) was rated on a 0 to 5 rating scale as described by Zhang et al. (2016). Briefly, disease severity of basal stems was scored on a 0 to 5 rating scale: $0=$ no noticeable infection; $1=$ diseased tissue occupies $\leq 25 \%$ of the cross-section; $2=$ diseased tissue occupies 25 to $50 \%$ of cross-section; $3=$ between 50 and $75 \%$ of the cross-section infected; $4=$ more than $75 \%$ of the cross-section infected; and $5=100 \%$ of cross-section diseased, plant dead.

Fungal isolation and inoculum preparation. Fungal isolation, purification, inoculum preparation, and maintenance were performed as described by Liban et al. (2016) with some modifications. All isolates were collected from the stem or stem base of infected canola stems. Briefly, infected stems were surface-sterilized in $50 \%(\mathrm{v} / \mathrm{v})$ bleach for $1 \mathrm{~min}$, rinsed with sterilized water, air dried, and cut into small pieces. These stem pieces were placed on nonclarified V8 agar juice medium $(200 \mathrm{ml}$ of $\mathrm{V} 8$ juice, $800 \mathrm{ml}$ water, $15 \mathrm{~g}$ agar, $0.75 \mathrm{~g}$ $\mathrm{CaCO}_{3}$ ) amended with $0.0035 \%$ (w/v) streptomycin sulfate and incubated under continuous florescent light at room temperature $\left(25^{\circ} \mathrm{C}\right)$ for 7 days. Each pure culture of $L$. maculans, obtained from a single spore, was transferred onto a new Petri plate containing V8 agar, as described in previous studies (Liban et al. 2016). Subsequently, each Petri plate was flooded with $3 \mathrm{ml}$ of sterile distilled water and L. $\mathrm{mac}$ ulans spores were harvested for pathogenicity testing under greenhouse conditions.

DNA extraction and PCR assays. Genomic DNA was extracted from pycnidiospores suspensions of purified L. maculans isolates using the method described by Liban et al. (2016). The presence or absence of avirulence $(A v r)$ alleles in L. maculans isolates collected from growers' field (2010 to 2015) was determined using PCR with primers that were specific to the six cloned Avr genes, derived from the original reports on cloning of the respective genes: AvrLm1 (Gout et al. 2006a), AvrLm2 (Ghanbarnia et al. 2015), AvrLmJ1/AvrLm5 (Van de Wouw et al. 2014; Balesdent and Howlett, unpublished data), AvrLm4-7 (Parlange et al. 2009), AvrLm6 (Fudal et al. 2007), and AvrLm11 (Balesdent et al. 2013). The presence of a PCR product indicated the presence of the avirulent allele, while the absence of the PCR product indicated that the avirulent allele was not present (Rong et al. 2015; Van de Wouw et al. 2010). For AvrLm4-7, two allelic variance of this avirulence gene interact with host $R \operatorname{lm} 4$ and $R \operatorname{lm} 7$ resistance genes where the presence and absence genotype indicates the presence and absence of $\operatorname{Avr} \operatorname{Lm} 7$; however, a single nucleotide change renders the gene virulent toward Rlm4. In the case of AvrLm4, an SNP marker would be needed to accurately determine the phenotype and for the purposes of this study, the pathogenicity assay was used to determine the frequency of the AvrLm4 allele. The distribution of two mating type alleles, MAT1-1 and MAT1-2, in L. maculans populations was also determined by using the multiplex PCR assay developed by Cozijnsen and Howlett (2003). In some cases, the PCR assay was repeated to verify results.

Pathogenicity tests. Avirulence profiles of $L$. maculans isolates were determined by inoculating the isolates onto a set of B. napus differentials listed in Table 1 (Zhang et al. 2016). Four cotyledon lobes of 7-day-old B. napus seedlings were wounded with modified tissue forceps to generate the route of infection. Each wounded site was

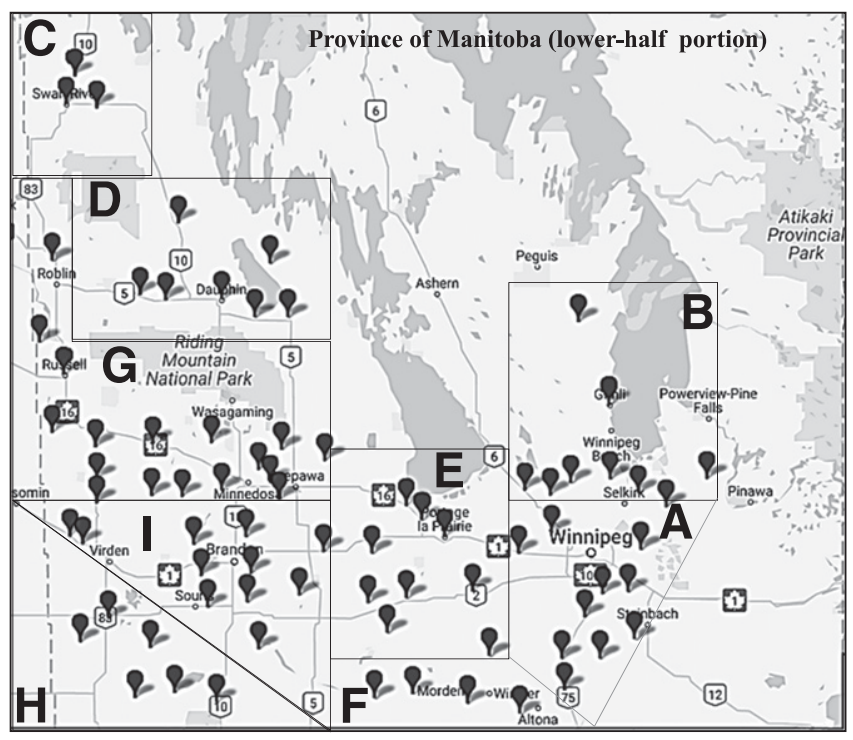

Fig. 1. Manitoba map of the nine regions in which blackleg infected canola stems were sampled. Sites are represented by letters and are as follows: A, Steinbach region; B, Selkirk area; C, Swan Valley; D, Dauphin area; E, Central Plains; F, Pembina Valley; G, Russell, Rossburn, and Roblin; H, Southwest area; I, Brandon area. Black pins in each site represent the area where fields were sampled. 
inoculated with a $10-\mu l$ droplet of the appropriate pycnidiospore suspension $\left(2 \times 10^{7}\right.$ spores $\left./ \mathrm{ml}\right)$. Disease symptoms on inoculated cotyledons were evaluated 14 days post inoculation (dpi) using a rating scale of 0 to 9 as described by Williams and Delwiche (1979). At least six plants of each differential variety were used for the pathogenicity test of each isolate. A total of 24 disease scores (four cotyledon lobes $\times$ six plants) were obtained for each isolate- $B$. napus interaction, and the average score was used to determine the interaction phenotype (IP), with avirulent IP scores ranging from 0 to 4.9 and virulent IP scores ranging from 5 to 9 (Liban et al. 2016). B. napus differentials used in this study (Table 1) enabled the identification of AvrLm1, AvrLm2, AvrLm3, AvrLm4, AvrLm7, AvrLm9, AvrLepR1, AvrLepR2, and AvrLmS. Surpass 400 (LepR3, RlmS) was used to identify AvrLmS in the population of fungal isolates collected. Since AvrLm1 interacts with LepR3 (Larkan et al. 2013), the presence of the AvrLmS allele could not be identified in isolates carrying AvrLm1. The race structure of each isolate was determined according to Balesdent et al. (2005). The distribution and frequency of AvrLm2, AvrLm3, AvrLm 9, AvrLepR1, AvrLepR2, and AvrLmS alleles within the fungal population were assessed using differential cultivars. The avirulence frequency of AvrLm5, AvrLm6, and AvrLm11 was assessed with PCR. The avirulence frequency of AvrLm1, AvrLm4, and AvrLm7 were assessed with both PCR and differential cultivars.

Data analysis. $L$. maculans isolates collected from all regions in a given year were evaluated as a population, and isolates collected from a given site in a given year was evaluated as a subpopulation. The isolates in each population and subpopulation were analyzed for the frequency of $A v r$ alleles. Genetic diversity of these L. maculans populations and subpopulations was studied individually. Simpson's index of diversity was calculated to estimate genetic diversity of the population (Balesdent et al. 2006). The Margalef index, which measures the richness in species and/or races of a population, was also calculated (Balesdent et al. 2006). The distribution of two mating alleles, MAT1-1 and MAT1-2, were determined in the L. maculans populations collected each year and in the subpopulations of isolates collected within each of the nine Manitoban regions (i.e., isolates from each region collected from 2010 to 2015 pooled together). The ratios between the two alleles were analyzed with a $\chi^{2}$ test (Ugoni and Walker 1995) to determine if the ratios varied from 1:1. A $\chi^{2}$ homogeneity test was also performed to determine if the mating type allele distribution in the collected isolates was similar among all years and regions. Because the significance level of $\alpha=0.05$ was used for testing the allele ratios, $\chi^{2} 95 \%$ (with degrees of freedom [df] corresponding to the number of samples -1 ) from the $\chi^{2}$ table was used to perform the $\chi^{2}$ homogeneity test.

\section{Results}

Disease incidence and severity of blackleg. The six-year survey of canola crops in Manitoba, Canada, from 2010 to 2015 has shown a general trend in blackleg incidence across nine regions, where the average disease incidence increases from 0 to $98 \%$ (Table 2). The Steinbach (A), Central Plains (E), Pembina Valley (F), and Roblin/Russel/

Table 1. Brassica napus varieties/lines used as differentials to identify avirulence genotypes of Leptosphaeria maculans isolates

\begin{tabular}{lll}
\hline Cultivar/line & \multicolumn{1}{c}{ R-genes } & \multicolumn{1}{c}{ Reference } \\
\hline MT29 & $R \operatorname{lm} 1,9$ & Delourme et al. 2008 \\
01-23-2-1 & $R \operatorname{lm} 7$ & Dilmaghani et al. 2009 \\
Quinta & $R \operatorname{lm} 1,3$ & Kutcher et al. 2010 \\
Surpass 400 & LepR3, RlmS & Larkan et al. 2013 \\
1065 & LepR1 & Kutcher et al. unpublished \\
1135 & LepR2 & Kutcher et al. unpublished \\
Glacier & Rlm 2,3 & Balesdent et al. 2001 \\
Jet Neuf & Rlm4 & Gout et al. 2006b \\
Goéland & Rlm9 & Balesdent et al. 2006 \\
Bristol & Rlm2, Rlm9 & Balesdent et al. 2005 \\
02-22-2-1 & Rlm3 & Gout et al. 2006b \\
Westar & No R gene & Balesdent et al. 2001 \\
\hline
\end{tabular}

Rossburn $(\mathrm{G})$ regions shared a similar pattern in terms of blackleg incidence where there was a continuous increase observed in each of these regions ranging from 0 to $98 \%, 0$ to $92 \%, 6$ to $84 \%$, and 5 to $65 \%$ (A, E, F, and G, respectively) from 2010 to 2015 (Table 2). The Swan Valley region (C) had the lowest range of blackleg incidence over the six years, ranging from 0 to $51 \%$. The Dauphin (D) region exhibited a range of 1 to $4 \%$ blackleg incidence in 2010 , and in 2013 the range of disease incidence increased up to $30 \%$, where it remained constant to 2015 (Table 2). The Southwest area $(\mathrm{H})$ exhibited a range of blackleg incidence of 1 to $17 \%$ in 2010 , increasing to $50 \%$ in 2011, and decreasing to 10 and $4 \%$ in 2012 and 2013, respectively. The Selkirk area (B) exhibited the exact pattern in terms of increase and decrease of blackleg incidence. The Brandon (I) area also exhibited a similar pattern, where the blackleg incidence in 2010 was relatively low ranging from 1 to $20 \%$, and in 2012 increased to a range up to 50\%, decreasing in 2013 and then increasing once again to a range of 13 to $55 \%$ blackleg incidence in 2014. However, unlike regions $\mathrm{H}$ and $\mathrm{B}$, the range of blackleg incidence increased up to $90 \%$ in 2015 (Table 2). Disease severity, rated on a 0 to 5 scale, ranged from 1.4 to 1.6 from 2010 to 2015, indicating very little change in severity from year to year within the nine regions of Manitoba sampled (data not shown).

Avirulent alleles in fungal populations. The presence or absence of AvrLm1, AvrLm2, AvrLm3, AvrLm4-7, AvrLm5, AvrLm6, AvrLm9, AvrLm11, AvrLepR1, AvrLepR2, and AvrLmS alleles was determined using $B$. napus differentials, alone or coupled with PCR assays, for each L. maculans collected in this six-year survey of canola in Manitoba. The frequency and distribution of the Avr alleles carried by the fungal isolates were first analyzed by year of sampling, with years representing populations of the pathogen (Fig. 2). From the total 956 L. maculans isolates collected in 2010 to 2015, seven Avr alleles, AvrLm2, AvrLm4, AvrLm5, AvrLm6, AvrLm7, Avrlm11, and AvrLmS, were the most abundantly identified and were present in 50 to $100 \%$ of the collected isolates over the six-year period (Fig. 2A). When each year was examined more closely, isolates collected in 2013 appeared to harbor lower frequency of AvrLm4, AvrLm5, AvrLm6, AvrLm7, AvrLm11, and AvrLmS alleles (63, 76, 66, 65, 77 , and $12 \%$, respectively; Fig. 2A). However, in 2015, the frequency of these alleles, with the exception of AvrLm5, was greater than in the other years of sampling $(77,99,99,96$, and $92 \%$, respectively; Fig. 2A). The AvrLm2 allele frequency, on the other hand, appeared to remain relatively similar in isolates collected in 2012 to $2015(67,63,64$, and $62 \%$, respectively; Fig. 2A).

Table 2. \% Range of blackleg incidence observed in each region per year of surveillance

\begin{tabular}{llccccc}
\hline & \multicolumn{6}{c}{ \% Range of blackleg incidence } \\
\cline { 2 - 7 } Region $^{\mathbf{b}}$ & $\mathbf{2 0 1 0}$ & $\mathbf{2 0 1 1}$ & $\mathbf{2 0 1 2}$ & $\mathbf{2 0 1 3}$ & $\mathbf{2 0 1 4}$ & $\mathbf{2 0 1 5}$ \\
\hline A & $0-10$ & $5-45$ & $1-30$ & $9-84$ & $7-98$ & $11-50$ \\
B & $0-5$ & $8-50$ & $1-11$ & $0-7$ & $11-16$ & $4-50$ \\
C & 0 & 2 & $\mathrm{ND}^{\mathbf{c}}$ & $1-6$ & 51 & $1-8$ \\
D & $1-4$ & $1-11$ & $2-19$ & $1-30$ & $8-30$ & $3-30$ \\
E & $0-10$ & $3-15$ & $3-50$ & $11-40$ & $10-92$ & $24-76$ \\
F & $6-40$ & $0-8$ & $5-21$ & $3-50$ & $15-84$ & $21-60$ \\
G & $5-14$ & $1-8$ & $1-30$ & $5-33$ & $2-65$ & $1-13$ \\
H & $1-17$ & $21-50$ & $0-10$ & $2-4$ & $5-30$ & $4-90$ \\
I & $1-20$ & $1-15$ & $4-50$ & $1-15$ & $13-55$ & $0-6$ \\
\hline
\end{tabular}

${ }^{a}$ Leptosphaeria maculans isolates were collected from canola crops obtained in nine Manitoba regions (as shown in Fig. 1): Areas are defined by letters and are as follows: A, Steinbach area; B, Selkirk area; C, Swan Valley; D, Dauphin; E, Central Plains; F, Pembina Valley; G, Roblin, Russell, Rossburn area; H, Southwest area; I, Brandon area.

${ }^{b}$ Incidence of blackleg evaluated based on the percentage of blackleg infected stems (stems with basal canker) in 100 collected crop samples/year/field within each region. Blackleg incidence $(\%)$ reported as the range of incidence for each region. Mean value and standard deviation was not calculated because crop samples collected for each year within each of the nine regions were not from the same fields (i.e., single replicate) due to crop rotation of canola by producers.

${ }^{\mathrm{c}}$ Not determined; no isolates obtained in this region. 
As shown in Figure 2B, the alleles that were detected the least abundantly in the 956 isolates collected from 2010 to 2015 were AvrLm1, AvrLm3, AvrLm9, AvrLepR1, and AvrLepR2. The AvrLm1 allele was detected in isolates collected in all six years and was relatively stable from 2010 to 2014 (15.2\% in $2010,16.4 \%$ in 2011 , $12.5 \%$ in 2012 and 2013, and $11.1 \%$ in 2014; Fig. 2B). In 2015, the AvrLm1 allele was detected at the highest observed frequency of $28 \%$. Interestingly, the AvrLm3 allele illustrated a pattern of decreasing frequency in the collected isolates from 2010 to 2014 (Fig. 2B), where the allele frequency decreased from $6.52 \%$ in 2010 to $4.92 \%$ in 2011 , to $1.79 \%$ in 2012 , to $0.7 \%$ in 2013 , and to $0 \%$ in 2014 . However, in 2015 , this allele was detected only in $1.3 \%$ of the 302 L. maculans isolates collected (Fig. 2B). The AvrLm 9 allele shared a similar decreasing pattern as was observed for Avrlm3, but the AvrLm9 allele was not detected in any isolates in 2010. From 2011, when the allele was detected, to 2013, the observed AvrLm 9 allelic frequency in isolates collected those years decreased from $3.2 \%$ in 2011 to $0.8 \%$ in $2012,0.7 \%$ in 2013, and $0 \%$ in 2014 (Fig. 2B). An intermittent frequency pattern from L. maculans isolates collected in 2010 to 2015 was observed in the AvrLepR2 allele, where it was not detected in isolates collected in 2010, 2012, or 2013, but was detected in isolates collected in 2011 (1.6\%), 2014 (6.7\%), and 2015 (0.3\%) (Fig. 2B). The AvrLepR1 allele frequency
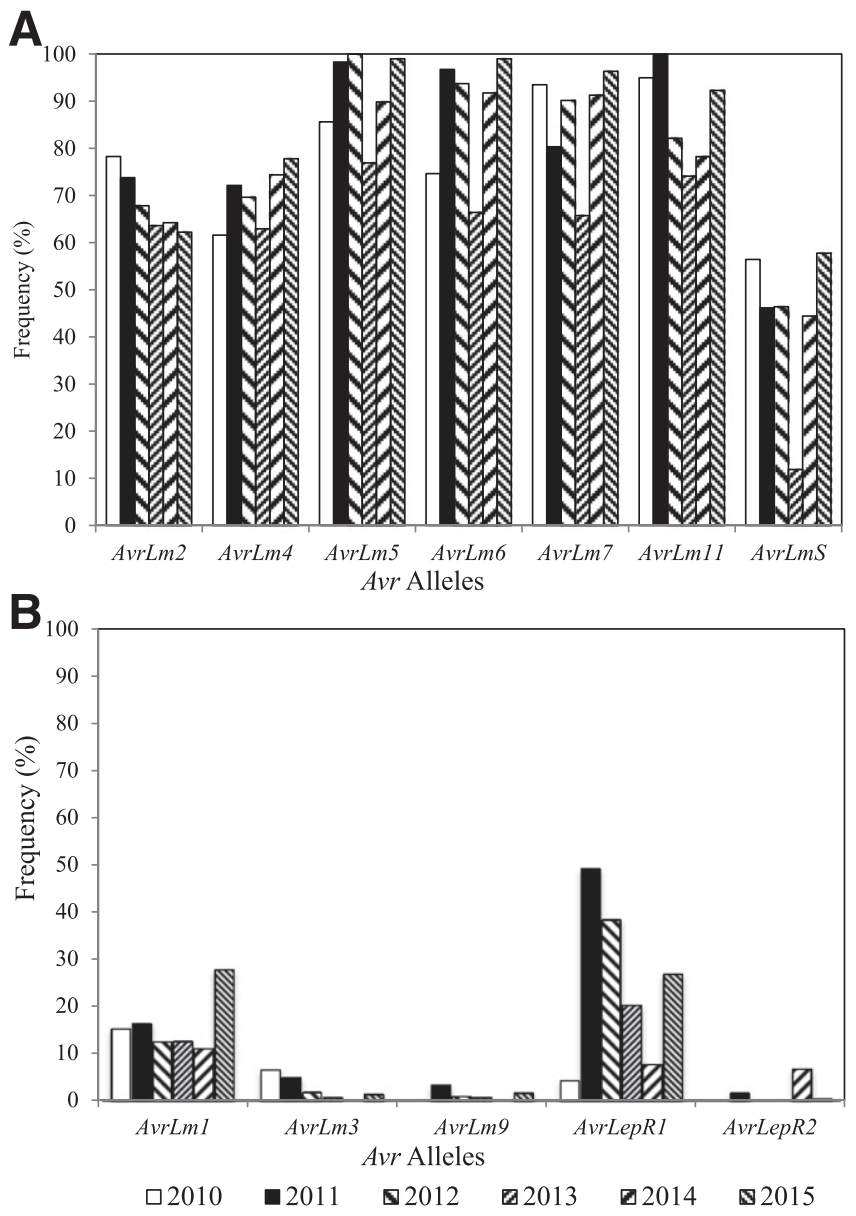

Fig. 2. Frequency of Avr alleles in Leptosphaeria maculans populations (2010 to 2015). A, The frequency of the most prevalent alleles (AvrLm2, AvrLm4, AvrLm5, AvrLm6, AvrLm7, AvrLm11, and AvrLmS) within the L. maculans population. B, Frequency of the least prevalent alleles (AvrLm1, AvrLm3, AvrLm9, AvrLepR1, and AvrLepR2). The frequency of the avirulence allele in each year was calculated based on the percentage of isolates carrying the avirulence allele among all isolates tested in that year. The presence of AvrLm1, AvrLm2, AvrLm4, and AvrLm7 was assessed using a combination of PCR and pathogenicity assays, while the presence of AvrLm5, AvrLm6, and AvrLm11 alleles were determined using PCR only. Presence of the other five Avr alleles was determined with pathogenicity assays only. observed from the collected isolates, on the other hand, appeared to fluctuate the most from year to year ( $4 \%$ of the 139 isolates collected in $2010,49 \%$ of the 61 isolates collected in $2011,38 \%$ of the 105 isolates collected in 2012, 20\% of the 143 isolates collected in $2013,7 \%$ of the 207 isolates collected in 2014, and $26 \%$ of the 302 isolates collected in 2015; Fig. 2B).

When the L. maculans isolates were analyzed by region of Manitoba in which canola crops were sampled, the detected frequency of AvrLm5, AvrLm6, AvrLm7, and AvrLm11 alleles were great in each region surveyed (Fig. 3A) with an average frequency of $90.2 \%( \pm 3 \%)$, $85 \%( \pm 8 \%), 86 \%( \pm 7 \%)$, and $81 \%( \pm 6 \%)$, respectively. Conversely, larger fluctuations of the AvrLm1, AvrLm4, and AvrLepR1 allele frequencies were observed among the nine Manitoban regions (Fig. $3 \mathrm{~A}$ and B). The average frequency of AvrLml was $15.4 \%( \pm 10 \%)$ and ranged from $3.8 \%$ in the Central Plains to $33.0 \%$ in Pembina Valley (Fig. 3B), while the average frequency of AvrLm4 was $70 \%( \pm 13 \%)$, ranging from $43.9 \%$ in the Southwest area to $88.4 \%$ in the Steinbach area (Fig. 3A). The average AvrLepR1 allele frequency within the nine regions was $16.8 \%( \pm 6 \%)$, ranging from $6 \%$ in the Swan Valley region to $24 \%$ in the Russell/Roblin/ Rossburn area (Fig. 3B). The less frequently detected AvrLm3, AvrLm9, and AvrLepR 2 alleles ranged from 0 to $7 \%$ across the nine regions (Fig. 3B).
A

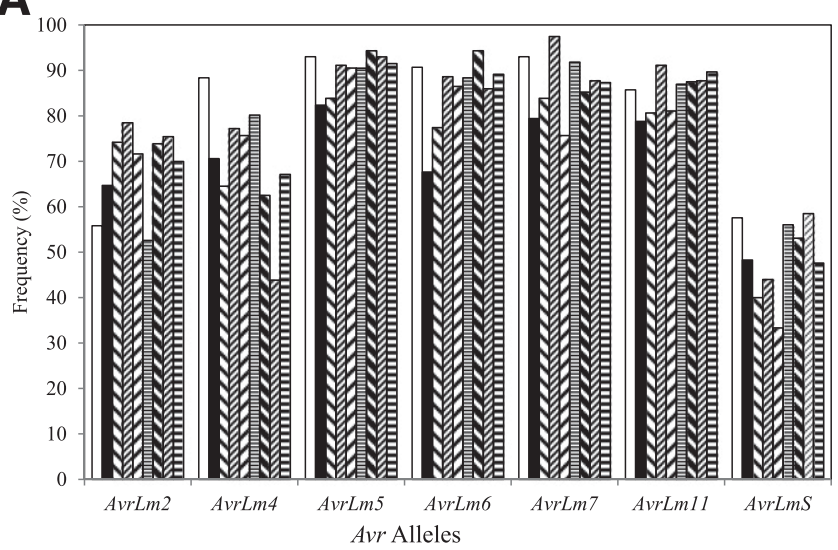

B

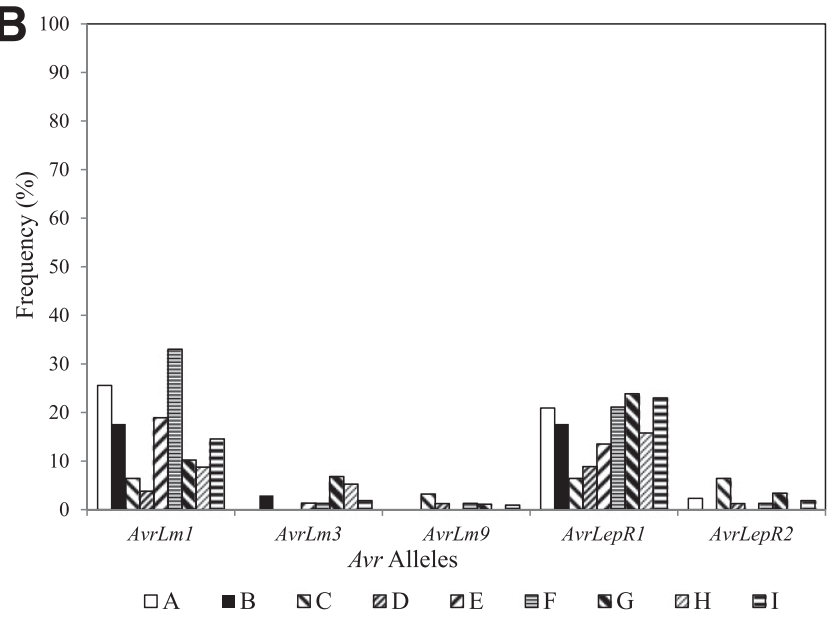

Fig. 3. Frequency of avirulence alleles in Leptosphaeria maculans populations from nine Manitoba regions (see below). A, The frequency of the most prevalent alleles (AvrLm2, AvrLm4, AvrLm5, AvrLm6, AvrLm11, and AvrLmS). B, Frequency of the least prevalent alleles (AvrLm1, AvrLm3, AvrLm9, AvrLepR1, and AvrLepR2) within the L. maculans population. The presence of AvrLm1, AvrLm2, AvrLm4, and AvrLm7 was assessed using a combination of PCR and pathogenicity assays, while the presence of AvrLm5, AvrLm6, and AvrLm11 were determined using PCR only. Presence of the other five Avr alleles was determined with pathogenicity assays only. Nine regions (Fig. 1) are as follows: A, Steinbach region; B, Selkirk area; C, Swan Valley; D, Dauphin area; E, Central Plains; F, Pembina Valley; G, Russell, Rossburn, and Roblin; H, Southwest area; I, Brandon area. 
Genetic diversity and race structure analysis in $L$. maculans populations. A total of 12 avirulence alleles were detected and analyzed for the L. maculans isolates collected from 2010 to 2015. A total of 27 race structures was identified for isolates of $L$. maculans collected and analyzed in 2010, 23 races from isolates collected in 2011, 53 from 2012 isolates, 62 from 2013 isolates, 59 from 2014 isolates, and 51 from 2015 isolates (Table 2). In total, 170 races of L. maculans were identified from 956 isolates collected from 2010 to 2015. The three most prevalent races were: Avr-2-4-5-6-7-11 detected in 143 isolates, Avr-2-4-5-6-7-11-(S) in 93 isolates, and Avr-1-4-5-6-7-11-(S) in 54 isolates (Fig. 4). Seven isolates were identified, with race structure of Avr null (no detectable avirulent alleles), Avr-1, Avr-4, Avr-5, Avr-7, and Avr-11. The race structure also appeared to be relatively stable from year to year in that a majority of the isolates collected each year had a race structure similar to Avr-2-4-5-6-11-(S) (Fig. 4), with some minor changes. For example, in 2011, AvrLepRl was prominent within the race structure while in 2014, AvrLml was identified more frequently within the race structure. Over the six years of the survey in Manitoba, there was a high level of race diversity detected in the L. maculans isolates. With exception of 2010, for which the isolates collected had a Simpson index of 0.77, Simpson index in isolates collected in the other five years was more than 0.90 , indicating high genetic diversity of populations each year (Table 3 ). The Margalef index values also indicated high race diversity in these $L$. maculans populations in Manitoba, ranging from 5.27 in 2010 to 5.35 in 2011, 11.02 in 2012, 12.29 in 2013, 10.88 in 2014, and 8.76 in 2015.

Genetic diversity and race structure analysis in $L$. maculans subpopulations. The L. maculans isolates race structure was also analyzed based on regions surveyed in Manitoba (Table 4). As shown in Table 4, the number of races within each region varied depending on the number of isolates collected within each region, with the greatest number of races detected in the Brandon and Pembina Valley regions (118 and 117 races, respectively). As shown in Table 5, the two most frequently observed races, in seven of the nine regions, were $A v r$ 2-4-5-6-7-11 and Avr-2-4-5-6-7-11-(S). The Selkirk, Southwest, and Pembina Valley areas, however, differed from other regions sampled. The top two races detected in Selkirk were Avr-2-4-5-7-11

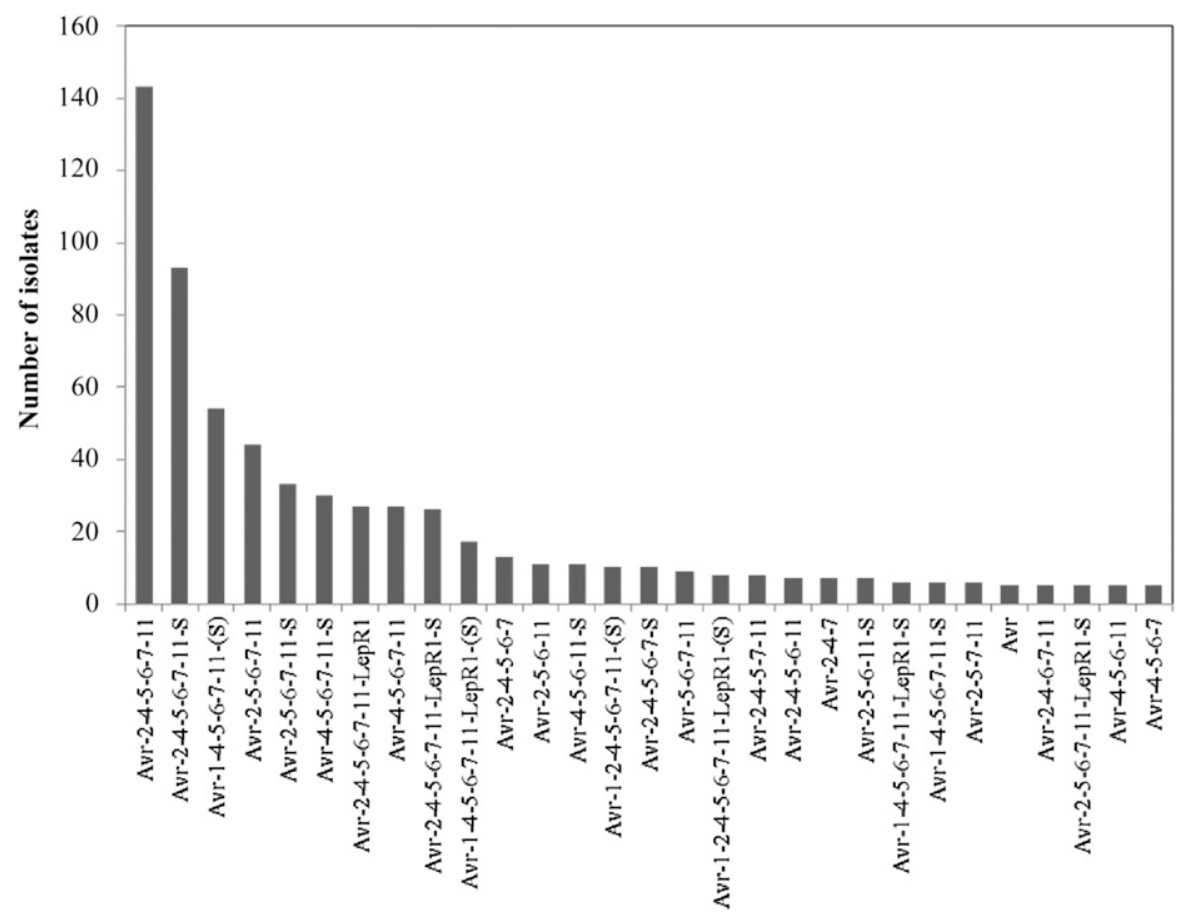

Fig. 4. Frequency of 29 major Leptosphaeria maculans races (each race with five or more isolates) based on their avirulence alleles. A total of $964 L$. maculans isolates were analyzed. It should be noted that the race "Avr" represents Avr-null.

Table 3. Avr allele diversity (i.e., races) of the collected Leptosphaeria maculans populations from 2010 to 2015 and the distribution of mating type alleles year to year

\begin{tabular}{|c|c|c|c|c|c|c|c|c|c|}
\hline Year & No. of isolates & No. of races & Margalef index ${ }^{a}$ & Simpson index ${ }^{b}$ & Number of fields & $M A T 1-1^{\mathrm{c}}$ & $M A T 1-2^{c}$ & $\chi^{2}$ & $P$ value \\
\hline 2010 & 138 & 27 & 5.27 & 0.77 & 68 & 54 & 84 & 6.52 & 0.01 \\
\hline 2011 & 61 & 23 & 5.35 & 0.93 & 55 & 27 & 34 & 0.80 & 0.44 \\
\hline 2012 & 105 & 53 & 11.02 & 0.97 & 54 & 53 & 52 & 0.009 & 1.00 \\
\hline 2013 & 143 & 62 & 12.29 & 0.95 & 74 & 78 & 65 & 1.18 & 0.32 \\
\hline 2014 & 207 & 59 & 10.88 & 0.95 & 97 & 115 & 92 & 2.56 & 0.13 \\
\hline \multirow[t]{2}{*}{2015} & 302 & 51 & 8.76 & 0.92 & 71 & 122 & 180 & 11.13 & 0.001 \\
\hline & & & & & & \multicolumn{2}{|c|}{$\chi^{2}$ for homogeneity } & 22.21 & 0.002 \\
\hline
\end{tabular}

${ }^{a}$ Margalef index was used to determine the race richness within each L. maculans population from year to year. The higher the value, the "richer" the race of a given population.

b Simpson's index was used to determine the allele race diversity within each $L$. maculans population from year to year. Values close to 1 indicate a high race diversity.

${ }^{c}$ Number of isolates carrying the MAT1-1 and MAT1-2 alleles as determined via PCR. Ratio between both mating types determined as illustrated by $\chi^{2}\left(\chi^{2}\right)$ where $P$ values less than 0.05 are significantly different than a $1: 1$ ratio.

${ }^{\mathrm{d}} \chi^{2}$ for homogeneity was determined to see whether the isolates collected from each year were behaving similarly in terms of mating type allele distribution (i.e., a 1:1 ratio between MAT1-1 and MAT1-2 alleles in all years). A $\chi^{2}$ homogeneity value of 22.21 is $>11.07(1-0.05 ; \mathrm{df}=5)$, which indicates that the mating type allele distribution among the $L$. maculans populations from year to year are different than a 1:1 ratio. 
and Avr-1-4-5-7-11-LepRI-(S) (9 and 6\% of the 34 isolates collected, respectively) while the top two races detected in the Southwest area were Avr-2-5-6-7-11-(S) and Avr-2-4-5-6-7-11-(S) (15 and $7 \%$ of the 73 isolates collected, respectively). Isolates collected from the Pembina Valley area showed a top two race structure of Avr-2-5-6-7-11 and Avr-1-4-5-6-7-11-(S) (14 and 11\% of the 250 isolates collected, respectively; Table 5).

Within the nine Manitoban regions sampled, there was a high level of race diversity detected in the collected L. maculans isolates. With the exception of the Steinbach, Selkirk, and Swan Valley, for which the isolates collected had Simpson indices of $0.75,0.88$, and 0.84 , respectively, the Simpson index in isolates collected in the other regions was $\geq 0.90$, indicating high genetic diversity within those regions (Table 4). The Margalef index values also indicated high race diversity in the L. maculans subpopulations in Manitoba, ranging from 6.41 to 21.50 (Table 4). Furthermore, the assortment of races found within each of the nine regions also illustrated high levels of diversity, where none of the regions sampled shared the exact same race structures.
Identification of mating type alleles in L. maculans populations and subpopulations. To better understand the long-term change of the pathogen populations, PCR products corresponding to one of the two mating type alleles were amplified from $956 \mathrm{~L}$. maculans isolates tested. Both mating type alleles were present in the populations within all nine Manitoban regions examined (Table 4), indicating that isolates with the two mating alleles were well adapted to field conditions in Manitoba, Canada, and that mating type did not restrict sexual recombination. Overall, 449 MAT1-1 alleles and 507 MAT1-2 alleles were identified from 897 isolates. The ratio of the two mating type alleles did not appear to deviate significantly from a 1:1 ratio in isolates collected from 2011 to 2014 (Table 3). However, in L. maculans isolates collected in 2010 and $2015, \chi^{2}$ values of $6.652(P=$ $0.013)$ and $11.13(P=0.001)$ were calculated, respectively, indicating that isolates collected within those years did not carry the MAT11 and MAT1-2 alleles in a 1:1 ratio. In fact, in both years the MAT1-2 allele was more frequently detected in the collected isolates. $\chi^{2}$ for homogeneity was also determined to see whether the isolates collected from each year were behaving similarly throughout the six

Table 4. Margalef index, Simpson index, and mating type alleles distribution in Leptosphaeria maculans subpopulations

\begin{tabular}{|c|c|c|c|c|c|c|c|c|c|}
\hline Regiona $^{a}$ & No. of isolates & No. of races & No. of fields & Margalef index ${ }^{b}$ & Simpson index & $M A T 1-1^{\mathrm{d}}$ & MAT1-2d & $\chi^{2}$ & $P$ value \\
\hline A & 43 & 29 & 17 & 7.44 & 0.75 & 27 & 16 & 2.81 & 0.13 \\
\hline B & 34 & 25 & 20 & 7.37 & 0.88 & 15 & 19 & 0.47 & 0.61 \\
\hline $\mathrm{C}$ & 31 & 23 & 18 & 6.41 & 0.84 & 11 & 20 & 2.61 & 0.15 \\
\hline D & 88 & 47 & 39 & 10.27 & 0.97 & 36 & 52 & 2.91 & 0.11 \\
\hline $\mathrm{E}$ & 94 & 48 & 33 & 10.34 & 0.95 & 46 & 48 & 0.04 & 0.92 \\
\hline $\mathrm{F}$ & 250 & 117 & 101 & 21.01 & 0.97 & 124 & 123 & 0.004 & 1.00 \\
\hline G & 113 & 65 & 49 & 13.54 & 0.96 & 43 & 70 & 6.45 & 0.01 \\
\hline $\mathrm{H}$ & 73 & 50 & 40 & 11.42 & 0.90 & 34 & 39 & 0.34 & 0.64 \\
\hline I & 230 & 118 & 102 & 21.50 & 0.96 & 113 & 117 & 0.07 & 0.84 \\
\hline & & & & & & \multicolumn{2}{|c|}{$\chi^{2}$ for homogeneity } & 15.71 & 0.003 \\
\hline
\end{tabular}

${ }^{a}$ L. maculans isolates were collected from canola crops obtained in nine Manitoba regions (as shown in Fig. 1): Areas are defined by letters and are as follows: A, Steinbach area; B, Selkirk area; C, Swan Valley; D, Dauphin; E, Central Plains; F, Pembina Valley; G, Roblin, Russell, Rossburn area; H, Southwest area; I, Brandon area.

b Margalef index was used to determine the race richness within each L. maculans population from year to year. The higher the value, the "richer" the race of a given population.

c Simpson's index was used to determine the allele race diversity within each $L$. maculans population from year to year. Values close to 1 indicate a high race diversity.

${ }^{\mathrm{d}}$ Number of isolates carrying the MAT1-1 and MAT1-2 alleles as determined via PCR. Ratio between both mating types determined as illustrated by $\chi^{2}\left(\chi^{2}\right)$ where $P$ values less than 0.05 are significantly different than a $1: 1$ ratio.

e $\chi^{2}$ for homogeneity was determined to see whether the isolates collected from each year were behaving similarly in terms of mating type allele distribution (i.e., a 1:1 ratio between MAT1-1 and MAT1-2 alleles). A $\chi^{2}$ homogeneity value of 15.71 is $>15.51(1-0.05$; df $=8)$, which indicates that the mating type allele distribution among the L. maculans populations from year to year are different from one another.

Table 5. The top five races identified in the collected L. maculans isolates from each of the nine Manitoban regions

\begin{tabular}{|c|c|c|c|c|c|}
\hline No. of isolates ${ }^{a}$ & $\operatorname{Region} \mathbf{A}^{\mathbf{b}}$ & No. of isolates & Region B & No. of isolates & Region C \\
\hline $8(18 \%)$ & $A v r-2-4-5-6-7-11-(S)$ & $3(9 \%)$ & $A v r-2-4-5-7-11$ & $8(26 \%)$ & Avr-2-4-5-6-7-11 \\
\hline $7(16 \%)$ & Avr-2-4-5-6-7-11 & $2(6 \%)$ & Avr-1-4-5-7-11-LepR1-(S) & $5(6 \%)$ & Avr-2-4-5-6-7-11-(S) \\
\hline $6(14 \%)$ & $A v r-1-4-5-6-7-11-(S)$ & $2(6 \%)$ & $A v r-2-4$ & $1(3 \%)$ & Avr-null \\
\hline $3(7 \%)$ & Avr-2-4-5-6-7-11-LepR1-(S) & $2(6 \%)$ & Avr-2-4-5-6-7-11 & $1(3 \%)$ & $A v r-1$ \\
\hline $2(5 \%)$ & Avr-4-5-6-7-11 & $2(6 \%)$ & Avr-2-4-5-6-7-11-LepR1-(S) & $1(3 \%)$ & Avr-1-2-4-7-9-LepR1-(S) \\
\hline No. of isolates & Region D & No. of isolates & Region E & No. of isolates & Region F \\
\hline $23(26 \%)$ & Avr-2-4-5-6-7-11-(S) & $25(27 \%)$ & Avr-2-4-5-6-7-11 & $35(14 \%)$ & Avr-2-4-5-6-7-11 \\
\hline $22(25 \%)$ & Avr-2-4-5-6-7-11 & $8(9 \%)$ & $A v r-2-4-5-6-7-11-(S)$ & $27(11 \%)$ & $A v r-1-4-5-6-7-11-(S)$ \\
\hline $5(6 \%)$ & Avr-2-5-6-7-11 & $8(9 \%)$ & Avr-2-5-6-11 & $13(5 \%)$ & Avr-2-4-5-6-7-11-LepR1 \\
\hline $3(3 \%)$ & $A v r-1-4-5-6-7-11-(S)$ & $3(3 \%)$ & Avr-1-4-5-6-7-11-(S) & $12(5 \%)$ & $A v r-2-4-5-6-7-11-(S)$ \\
\hline $3(\%)$ & $A v r-2-5-6-7-11-(S)$ & $3(3 \%)$ & Avr-2-4-5-6-7 & $11(4 \%)$ & Avr-4-5-6-7-11 \\
\hline No. of isolates & Region G & No. of isolates & Region $\mathrm{H}$ & No. of isolates & Region I \\
\hline $15(13 \%)$ & Avr-2-4-5-6-7-11 & $11(15 \%)$ & Avr-2-5-6-7-11-(S) & $27(12 \%)$ & Avr-2-4-5-6-7-11-(S) \\
\hline $13(12 \%)$ & $A v r-2-4-5-6-7-11-(S)$ & $5(7 \%)$ & Avr-2-4-5-6-7-11-(S) & $18(8 \%)$ & Avr-2-4-5-6-7-11 \\
\hline $9(8 \%)$ & $A v r-2-5-6-7-11-(S)$ & $5(7 \%)$ & Avr-2-4-5-7-11 & $17(7 \%)$ & Avr-2-5-6-7-11 \\
\hline $5(4 \%)$ & $A v r-1-4-5-6-7-11-(S)$ & $4(5 \%)$ & Avr-2-5-6-7-11-LepR1-(S) & $17(7 \%)$ & $A v r-2-5-6-7-11-(S)$ \\
\hline $5(4 \%)$ & Avr-2-4-5-6-7-11-LepR1-(S) & $3(4 \%)$ & Avr-2-4-5-6-7-11-LepR1 & $10(4 \%)$ & Avr-2-4-5-6-7-11-LepR1-(S) \\
\hline
\end{tabular}

${ }^{a}$ Number of each isolate collected within the region that harbors the race structure with percentage of the total collected isolates from each region represented in brackets.

$\mathrm{b}$ The following letters represent the following Manitoba regions with total isolates collected in each of those regions in brackets: A, Steinbach area (43); B, Selkirk area (34); C, Swan Valley (31); D, Dauphin (88); E, Central Plains (94); F, Pembina Valley (250); G, Roblin, Russell, Rossburn area (113); H, Southwest area (73); I, Brandon area (230). 
years in terms of mating type allele distribution (i.e., a 1:1 ratio between MAT1-1 and MAT1-2 alleles in all years). As shown in Table 3, the $\chi^{2}$ homogeneity value calculated was 22.21 , which is $>11.07$ $(1-0.05 ; \mathrm{df}=5)$. This indicates that the mating type allele distribution of the L. maculans populations collected in 2010 to 2015 are not behaving similarly from year to year.

When the mating type distribution in the L. maculans subpopulation (i.e., by region) was analyzed by $\chi^{2}$ test, the mating type ratio in isolates collected from eight of the nine regions did not appear to deviate from a 1:1 ratio (Table 4). However, in isolates collected from the Roblin, Russell, Rossburn area, a $\chi^{2}$ value of $6.45(P=0.01)$ was calculated for that particular region, indicating that the isolates collected from this region appear to deviate from carrying the mating type alleles in a 1:1 ratio. In addition, the MAT1-2 allele appears to be harbored more frequently in isolates collected from this region. $\chi^{2}$ for homogeneity was determined to see whether the isolates collected from each region were behaving similarly from region to region in terms of mating type allele distribution (i.e., a 1:1 ratio between MAT1-1 and MAT1-2 alleles). As shown in Table 4, a $\chi^{2}$ homogeneity value of 15.71 was calculated, which is $>15.51$ ( $1-0.05$; $\mathrm{df}=8$ ). This indicates that the mating type allele distribution of the L. maculans subpopulation from region to region are behaving differently.

\section{Discussion}

This study reports on avirulence (Avr) and mating type allele frequency in L. maculans isolates collected from canola fields across nine regions in Manitoba, Canada, over a six-year period from 2010 to 2015. The presence of the AvrLm1, AvrLm2, AvrLm3, AvrLm4, AvrLm7, AvrLm5, AvrLm6, AvrLm9, AvrLm11, AvrLepR1, AvrLepR2, and the AvrLmS alleles was determined in 956 L. maculans isolates collected from 2010 to 2015 through pathogenicity testing, PCR, or a combination thereof. The PCR markers used for the three avirulence alleles without corresponding pathogenicity testing in this study (AvrLm5, AvrLm6, and AvrLm11) were shown to correspond to the phenotypic response as described in the cloning of the respective genes AvrLmJ1/AvrLm5 (Van de Wouw et al. 2014; Balesdent and Howlett, unpublished data), AvrLm6 (Fudal et al. 2007), and AvrLm11 (Balesdent et al. 2013). Van de Wouw et al. (2010) also illustrated that a positive PCR product was correlated with the presence of AvrLm1 and AvrLm6 avirulence alleles and that large deletions in these genes, rendering them virulent, could be distinguished (Van de Wouw et al. 2010).

The results obtained from these assays indicated that the most predominant alleles identified in the collected $L$. maculans isolates each year were AvrLm2, AvrLm4, AvrLm5, AvrLm6, AvrLm7, AvrLm11, and $A v r L m S$ alleles. It has been reported that L. maculans isolates collected from 2010 and 2011 in western Canada (Alberta, Saskatchewan, and Manitoba) shared a similar genotype, where more than $85 \%$ of the isolate population carried both AvrLm6 and AvrLm7 (Liban et al. 2016). AvrLm1 was also detected at low frequencies in the study by Liban et al. (2016), which is similar to the findings in this study. Here, the AvrLml allele was identified in less than $28 \%$ of the isolates collected, and the frequency of this allele was highly variable among the nine Manitoban regions ranging from $3.8 \%$ in the Dauphin area to $33 \%$ in the Pembina Valley. There are likely influencing factors that attribute to the variability observed in the AvrLm1 allele frequency in the L. maculans isolates collected from region to region. Agronomic practices, crop rotation, and selection pressure of $R l m$ genes carried in the host likely play an influential role in shaping the $A v r$ allele profiles and contributing to the diversity of $A v r$ alleles in the L. maculans isolates from region to region. It should also be noted that samples for this study were not obtained from the same field each year; blackleg infected canola crop stems from different fields within the same nine regions were collected and used to isolate L. maculans, which would also add variation to the frequency of $A v r$ alleles.

From 2010 to 2015 , the L. maculans isolates collected within each of the nine regions of Manitoba harbored the AvrLm9, AvrLepR2, and AvrLm3 alleles less abundantly. When examining each of the nine regions, the Dauphin area appeared to harbor isolates carrying AvrLm 9 and AvrLepR 2 alleles in less than $2 \%$ of the collected fungal population, while AvrLm3 was not detected in any of the isolates tested within this region. The AvrLm3 allele was not detected in isolates collected from crops in the Steinbach or Swan Valley regions, and identified in less than $2 \%$ of the population in isolates collected from the Central Plains, Pembina Valley, and Brandon areas. These findings are supported by the study conducted by Liban et al. (2016), where L. maculans isolates collected from Alberta, Saskatchewan, and Manitoba carried AvrLm3, AvrLm9, and AvrLepR2 in less than $10 \%$ of the fungal population. A previous study conducted by Zhang et al. (2016) suggested that $R \operatorname{lm} 3$ was one of the most frequently used $R$ genes in Canadian B. napus canola crop varieties. Consequently, the decrease in the frequency of the AvrLm3 allele from 2010 to 2014 observed in this study may be explained by the strong selection pressure exerted by the presence of the $R \operatorname{lm} 3$ gene in Canadian canola varieties. In a study conducted by Liban et al. (2016), all collected L. maculans isolates (in western Canada) that carried the AvrLm3 allele also carried AvrLm9. The authors suggested that the decline in AvrLm 9 frequency observed in this study may be due to negative selection against the $A v r L m 3$ allele and a potential linkage between $A v r L m 3$ and AvrLm9. Although it was not verified in this study if all collected isolates carrying AvrLm3 also carried AvrLm9, a linkage between the alleles may explain why both alleles are found in such low frequencies.

A hide and seek relationship between AvrLm3 and AvrLm4-7 was reported by Plissonneau et al. (2016), where L. maculans isolates carrying AvrLm4-7 also carried AvrLm3 (but the function of AvrLm3 was blocked by AvrLm4-7). A very recent paper by Plissonneau et al. (2017) demonstrated the "camouflaged model," where the loss of $R \operatorname{lm} 3$-mediated resistance response in isolates virulent toward $R \operatorname{lm} 3$ occurred by two methods. The first method was through deletion or inactivation mutations of AvrLm4-7, which resulted in virulent isoforms of the AvrLm3 protein. The second method was through point mutations in AvrLm4-7, which allowed for suppression of the $A v r L m 3$ phenotype to be maintained. The identification of three race structures of Avr-2-3-5-6-7-11-LepR1-(S) and Avr-2-3-56-7-9-11-LepR1-S within the L. maculans population in this study suggests that other factors are influencing this interaction. The AvrLm3 gene was recently cloned (Plissonneau et al. 2017), and collected L. maculans isolates from 2015 were subjected to PCR assays using AvrLm3 specific primers. Of the 302 isolates collected in 2015, 288 isolates carried the AvrLm3 allele despite the low frequency of the AvrLm3 phenotype reported in this study. This supports the "camouflage" model (Plissonneau et al. 2017) since the majority of these isolates also carried AvrLm4-7, which was reported to mask the AvrLm3 recognition by host Rlm3.

The race structure of the 956 isolates collected from 2010 to 2015 were evaluated, and three races, Avr-2-4-5-6-7-11, Avr-2-4-5-6-711-(S), and Avr-1-4-5-6-7-11-(S), were highly dominant. These findings indicate an uneven pathogen population, while the 170 races detected emphasizes the diversity. The stability of two major races Avr-2-4-5-6-7-11 and Avr-2-4-5-6-7-11-(S) within seven of the nine regions in the $L$. maculans population over the six-year study period points toward lower rates of sexual recombination. Early studies in western Canada indicated that pycnidiospores were the major source of inoculum for L. maculans infection and reported a lack of sexual reproduction in the L. maculans populations (Dilmaghani et al. 2009; Ghanbarnia et al. 2011; Guo et al. 2005). However, there have been no large-scale analysis of mating type alleles in Canadian L. maculans populations. The current data in this study illustrates that MAT1-1 and MAT1-2 alleles in two of the six years as well as two of the nine regions (in all years) were significantly different from a 1:1 ratio, indicating that sexual reproduction may be restricted in some regions. Interestingly, the dominant stable race in the collected $L$. maculans isolates also suggests that the fungal population primarily undergoes asexual reproduction within Manitoba. However, there were some regions that did exhibit an equal distribution of both mating type alleles, illustrating that sexual reproduction may not be limited to these regions in Manitoba but that other environmental factors may attribute to L. maculans reproductive lifecycle. 
The genetic diversity and complexity of the L. maculans population in western Canada has changed over time. Chen and Fernando (2006) reported the prevalence of PG2 isolates (carrying AvrLm2 and/or AvrLm3; carrying AvrLm1 and/or AvrLm4) in central Canada. Dilmaghani et al. (2009) reported an AvrLm3 and AvrLm9 allele frequency of $56.4 \%$ in collected fungal isolates in western Canada. However, the frequency in which the AvrLm3 and AvrLm 9 allele was detected in L. maculans isolates appeared to decrease to $1.5 \%$ in recent years as reported by Liban et al. (2016). In our study, a total of 170 races were identified from 956 L. maculans isolates. Interestingly, the considerably diverse number of races found within each region in L. maculans suggests the potential for blackleg to affect canola production in Manitoba because each resistance gene examined in the study can be overcome by at least one of the pathogen races detected. In addition, isolates with race structure Avr null, Avr-1, Avr-4, Avr-5, Avr-7, and Avr-11 were also detected in this study. Isolates with these race structures would contribute greatly to the disease progression as these isolates would likely be virulent toward most of the commercially available cultivars grown by local farmers. Simpson's evenness index, which measures relative frequency of races, were all relatively close to 1 throughout the years, suggesting a high risk of resistance breakdown within these regions of Manitoba. In addition, the pattern of increased disease incidence across the nine regions over the six-year period also suggested a potential breakdown in resistance; however, further studies are required to determine if there is an actual correlation between disease incidence and $A v r$ allele frequency and if other factors such as weather conditions or changes in cropping rotations are contributing factors. The results reported here describe the avirulence allele profile of L. maculans populations in Manitoba, Canada. The regional Avr allele distribution observed in the L. maculans isolates collected in this study validates the notion that a single specific resistance gene may not provide effective control of blackleg across Manitoba. Therefore, it is necessary to understand the Avr genotype in the pathogen and the relationship between races and different resistance genes under selection pressure, as these are primarily the most important factors to consider when breeding for blackleg resistance in Canada.

\section{Acknowledgments}

We would like to thank the Manitoba Canola Growers Association for supporting this research and providing access to canola fields. We also appreciate the technical support from Tom Henderson and Danny Hausermann at AAFC-Brandon, Manitoba Agriculture staff from across the province, and numerous summer students who provided us with a collection of canola stubble and disease survey data each year.

\section{Literature Cited}

Ansan-Melayah, D., Balesdent, M. H., Delourme, R., Pilet, M. L., Tanguy, X., Renard, M., and Rouxel, T. 1998. Genes for race-specific resistance against blackleg disease in Brassica napus L. Plant Breed. 117:373-378.

Balesdent, M. H., Attard, A., Ansan-Melayah, D., Delourme, R., Renard, M., and Rouxel, T. 2001. Genetic control and host range of avirulence toward Brassica napus cultivars Quinta and Jet Neuf in Leptosphaeria maculans. Phytopathology 91:70-76.

Balesdent, M. H., Barbetti, M. J., Li, H., Sivasithamparam, K., Gout, L., and Rouxel, T. 2005. Analysis of Leptosphaeria maculans race structure in a worldwide collection of isolates. Phytopathology 95:1061-1071.

Balesdent, M. H., Fudal, I., Ollivier, B., Bally, P., Grandaubert, J., Eber, F., Chèvre, A. M., Leflon, M., and Rouxel, T. 2013. The dispensable chromosome of Leptosphaeria maculans shelters an effector gene conferring avirulence towards Brassica rapa. New Phytol. 198:887-898.

Balesdent, M. H., Louvard, K., Pinochet, X., and Rouxel, T. 2006. A large-scale survey of races of Leptosphaeria maculans occurring on oilseed rape in France. Eur. J. Plant Pathol. 114:53-65.

Brun, H., Chevre, A. M., Fitt, B. D. L., Powers, S., Besnard, A. L., Ermel, M., Huteau, V., Marquer, B., Eber, F., Renard, M., and Andrivon, D. 2010. Quantitative resistance increases the durability of qualitative resistance to Leptosphaeria maculans in Brassica napus. New Phytol. 185:285-299.

Chen, Y., and Fernando, W. G. D. 2006. Prevelance of pathogenicity groups of Leptosphaeria maculans in Western Canada and North Dakota, USA. Can. J. Plant Pathol. 28:533-539.

Cozijnsen, A. J., and Howlett, B. J. 2003. Characterisation of the mating-type locus of the plant pathogenic ascomycete Leptosphaeria maculans. Curr. Genet. 43: 351-357.
Daverdin, G., Rouxel, T., Gout, L., Aubertot, J. N., Fudal, I., Meyer, M., Parlange, F., Carpezat, J., and Balesdent, M. H. 2012. Genome structure and reproductive behaviour influence the evolutionary potential of a fungal phytopathogen. PLoS Pathog 8:e1003020.

Delourme, R., Brun, H., Ermel, M., Lucas, M. O., Vallee, P., Domin, C., Walton, G., Li, H., Sivasithamparam, K., and Barbetti, M. J. 2008. Expression of resistance to Leptosphaeria maculans in Brassica napus double haploid lines in France and Australia is influenced by location. Ann. Appl. Biol. 153:259-269.

Delourme, R., Chèvre, A. M., Brun, H., Rouxel, T., Balesdent, M. H., Dias, J. S. Salisbury, P., Renard, M., and Rimmer, S. R. 2006. Major gene and polygenic resistance to Leptosphaeria maculans in oilseed rape (Brassica napus). Eur. J. Plant Pathol. 114:41-52.

Dilmaghani, A., Balesdent, M. H., Didier, J. P., Wu, C., Davey, J., Barbetti, M. J., Li, H., Moreno-Rico, O., Phillips, D., Despeghel, J. P., Vincenot, L., Gout, L., and Rouxel, T. 2009. The Leptosphaeria maculans - Leptosphaeria biglobosa species complex in the American continent. Plant Pathol. 58:1044-1058.

Eber, F., Lourgant, K., and Brun, H. 2011. Analysis of Brassica nigra chromosomes allows identification of a new effective Leptosphaeria maculans resistance gene introgressed in Brassica napus. Pages 5-9 in: Proceeding of the 13th International Rapeseed Congress, Prague.

Fitt, B. D. L., Brun, H., Barbetti, M. J., and Rimmer, S. R. 2006. World-wide importance of phoma stem canker (Leptosphaeria maculans and L. biglobosa) on oilseed rape (Brassica napus). Eur. J. Plant Pathol. 114:3-15.

Fudal, I., Ross, S., Gout, L., Blaise, F., Kuhn, M. L., Eckert, M. R., Cattolico, L., Bernard-Samain, S., Balesdent, M. H., and Rouxel, T. 2007. Heterochromatin-like regions as ecological niches for avirulence genes in the Leptosphaeria maculans genome: map-based cloning of AvrLm6. Mol. Plant-Microbe Interact. 20:459-470.

Ghanbarnia, K., Fernando, W. G. D., and Crow, G. 2011. Comparison of disease severity and incidence at different growth stages of naturally infected canola plants under field conditions by pycnidiospores of Phoma lingam as a main source of inoculum. Can. J. Plant Pathol. 33:355-363.

Ghanbarnia, K., Fudal, I., Larkan, N. J., Links, M. G., Balesdent, M. H., Profotova, B., Fernando, W. G. D., Rouxel, T., and Borhan, M. H. 2015. Rapid identification of the Leptosphaeria maculans avirulence gene AvrLm2, using an intraspecific comparative genomics approach. Mol. Plant Pathol. 16:699-709.

Gout, L., Eckert, M., and Rouxel, T. 2006a. Genetic variability and distribution of mating type alleles in field populations of Leptosphaeria maculans from France. Appl. Environ. Microbiol. 72:185-191.

Gout, L., Fudal, I., Kuhn, M. L., Blaise, F., Eckert, M., Cattolico, L., Balesdent, M. H., and Rouxel, T. 2006b. Lost in the middle of nowhere: the AvrLm 1 avirulence gene of the Dothideomycete Leptosphaeria maculans. Mol. Microbiol. 60:67-80.

Guo, X. W., Fernando, W. G. D., and Entz, M. 2005. Effects of crop rotation and tillage on blackleg disease of canola. Can. J. Plant Pathol. 27:53-57.

Jestin, C., Lode, M., Vallee, P., Domin, C., Falentin, C., Horvais, R., Coedel, S., Manzaneres-Dauleux, M. J., and Delourme, R. 2011. Association mapping of quantitative resistance for Leptosphaeria maculans in oilseed rape (Brassica napus L.). Mol. Breed. 27:271-287.

Kutcher, H. R., Balesdent, M. H., Rimmer, S. R., Rouxel, T., Chèvre, A. M., Delourme, R., and Brun, H. 2010. Frequency of avirulence genes in Leptosphaeria maculans in western Canada. Can. J. Plant Pathol. 32:77-85.

Kutcher, H. R., Brandt, S. A., Smith, E. G., Ulrich, D., Malhi, S. S., and Johnston, A. M. 2013. Blackleg disease of canola mitigated by resistant cultivars and fouryear crop rotations in western Canada. Can. J. Plant Pathol. 35:209-221.

Larkan, N. J., Lydiate, D. J., Parkin, I. A. P., Nelson, M. N., Epp, D. J., Cowling, W. A., Rimmer, S. R., and Borhan, M. H. 2013. The Brassica napus blackleg resistance gene LepR3 encodes a receptor-like protein triggered by the Leptosphaeria maculans effector AVRLM1. New Phytol. 197:595-605.

Larkan, N. J., Ma, L., and Borhan, M. H. 2015. The Brassica napus receptor-like protein RLM2 is encoded by a second allele of the LepR3/Rlm2 blackleg resistance locus. Plant Biotechnol. J. 13:983-992.

Liban, S. H., Cross, D. J., Kutcher, H. R., Peng, G., and Fernando, W. G. D. 2016. Race structure and frequency of avirulence genes in the western Canadian Leptosphaeria maculans pathogen population, the causal agent of blackleg in Brassica species. Plant Pathol. 65:1161-1169.

Long, Y., Wang, Z., Sun, Z., Fernando, W. G. D., McVetty, P. B. E., and Li, G. 2011. Identification of two blackleg resistance genes and fine mapping of one of these two genes in a Brassica napus canola cultivar 'Surpass 400'. Theor. Appl. Genet. 122:1223-1231.

Parlange, F., Daverdin, G., Fudal, I., Kuhn, M. L., Balesdent, M. H., Blaise, F., Grezes-Besset, B., and Rouxel, T. 2009. Leptosphaeria maculans avirulence gene AvrLm4-7 confers a dual recognition specificity by the Rlm4 and Rlm7 resistance genes of oilseed rape, and circumvents $R l m 4$-mediated recognition through a single amino acid change. Mol. Microbiol. 71:851-863.

Plissonneau, C., Blaise, F., Ollivier, B., Leflon, M., Carpezat, J., Rouxel, T., and Balesdent, M.-H. 2017. Unusual evolutionary mechanisms to escape effectortriggered immunity in the fungal phytopathogen Leptosphaeria maculans. Mol. Ecol. 26:2183-2198.

Plissonneau, C., Daverdin, G., Ollivier, B., Blaise, F., Degrave, A., Fudal, I., Rouxel, T., and Balesdent, M. H. 2016. A game of hide and seek between avirulence genes AvrLm4-7 and AvrLm3 in Leptosphaeria maculans. New Phytol. 209:1613-1624.

Rimmer, S. R. 2006. Resistance genes to Leptosphaeria maculans in Brassica napus. Can. J. Plant Pathol. 28 (S1):S288-S297. 
Rong, S., Feng, J., Li, Q., Fei, W., Ahmed, H. U., Liang, Y., Hwang, S. F., and Strelkov, S. E. 2015. Pathogenic variability and prevalence of $A v r$ genes in Leptosphaeria maculans populations from Alberta, Canada. J. Plant Dis. Prot. 122:161-168.

Rouxel, T., Grandaubert, J., Hane, J. K., Hoede, C., van de Wouw, A. P., Couloux, A., Dominguez, V., Anthouard, V., et al. 2011. Effector diversification within compartments of the Leptosphaeria maculans genome affected by RepeatInduced Point mutations. Nat. Commun. 2:202.

Rouxel, T., Penaud, A., Pinochet, X., Brun, H., and Gout, L. 2003. A 10-year survey of populations of Leptosphaeria maculans in France indicates a rapid adaptation towards the Rlml resistance gene of oilseed rape. Eur. J. Plant Pathol. 109:871-881.

Soyer, J. L., El Ghalid, M., Glaser, N., Ollivier, B., Linglin, J., Grandaubert, J., Balesdent, M. H., Connolly, L. R., Freitag, M., Rouxel, T., and Fudal, I. 2014. Epigenetic control of effector gene expression in the plant pathogenic fungus Leptosphaeria maculans. PLoS Genet. 10:e1004227.

Sprague, S. J., Balesdent, M. H., Brun, H., Hayden, H. L., Marcroft, S. J., Pinochet, X., Rouxel, T., and Howlett, B. J. 2006. Major gene resistance in Brassica napus (oilseed rape) is overcome by changes in virulence of populations of Leptosphaeria maculans in France and Australia. Eur. J. Plant Pathol. 114:33-40.

Ugoni, A., and Walker, B. F. 1995. THE CHI SQUARE TEST: An Introduction. COMSIG Rev. 4:61-64.
Van de Wouw, A. P., Cozijnsen, A. J., Hane, J. K., Brunner, P. C., McDonald, B. A., Oliver, R. P., and Howlett, B. J. 2010. Evolution of linked avirulence effectors in Leptosphaeria maculans is affected by genomic environment and exposure to resistance genes in host plants. PLoS Pathog 6:e1001180.

Van de Wouw, A. P., Lowe, R. G. T., Elliott, C. E., Dubois, D. J., and Howlett, B. J. 2014. An avirulence gene, AvrLmJ1, from the blackleg fungus, Leptosphaeria maculans, confers avirulence to Brassica juncea cultivars. Mol. Plant Pathol. 15:523-530.

Van de Wouw, A. P., Marcroft, S. J., Barbetti, M. J., Hua, L., Salisbury, P. A. Gout, L., Rouxel, T., Howlett, B. J., and Balesdent, M. H. 2009. Dual control of avirulence in Leptosphaeria maculans towards a Brassica napus cultivar with "sylvestris-derived" resistance suggests involvement of two resistance genes. Plant Pathol. 58:305-313.

Williams, P. H., and Delwiche, P. A. 1979. Screening for resistance to blackleg of crucifers in the seedling stage. Pages 164-170 in: Proceedings of a Eucarpiaconference on the breeding of cruciferous crops, Wageningen. The Netherlands.

Zhang, X., Peng, G., Kutcher, H. R., Balesdent, M. H., Delourme, R., and Fernando, W. G. D. 2016. Breakdown of Rlm3 resistance in the Brassica napus-Leptosphaeria maculans pathosystem in western Canada. Eur. J. Plant Pathol. 145:659-674. 